Nutzerinnen und Nutzer von digitalen Speichermedien sind für Maßnahmen zum Schutz der von ihnen gespeicherten personenbezogenen Daten verantwortlich. Die DIGITTRADE-Festplatte HDD HS256S unterstützt sie wirkungsvoll durch

v vollständige Verschlüsselung der Festplatte (256 Bit AES, CBCModus),

- Zwei-Faktor-Authentifizierung: Chipkarte und PIN (Besitz und Wissen),

- Verwaltung des Verschlüsselungsschlüssels (Erstellen, Ändern, Kopieren und Zerstören),

- externe Speicherung des Verschlüsselungsschlüssels (auf der Chipkarte).

Die Prüfung wurde durch die Sachverständigen Stephan HansenOest (rechtlich) und Andreas Bethke (technisch) vorgenommen.

Informationen zur Zertifizierung können abgerufen werden unter https://www.datenschutzzentrum.de/guetesiegel/register.htm\#130303 sowie https://www.european-privacy-seal.eu/awarded-seals/DE130030

\section{Sicherheitskarte mit One-Time Password und LED-Display}

Auf der CeBIT 2013 haben die Bundesdruckerei GmbH und die Infineon Technologies AG eine neue Sicherheits-Chipkarte mit LED Display und „One-Time Password" vorgestellt. Grundlage der neuen Technologie ist ein Sicherheits-Chip in der Karte, der bei jeder Transaktion ein einmaliges Passwort generiert und auf dem integrierten LED-Display anzeigt. Die Abfrage des "One-Time Password“ zusätzlich zum statischen Passwort erhöht die Sicherheit von Authentifizierungs- und Zahlungsapplikationen und schützt beispielsweise vor Angriffen auf Firmennetzwerke oder vor Kartenmanipulationen.

Ziel der seit 2011 bestehenden Innovationspartnerschaft von Bundesdruckerei und Infineon ist es, Technologien für die nächste Generation von Wert- und Sicherheits-dokumenten wie beispielweise Betriebsausweise oder elektronische Identitätsdokumente (eID) zu entwickeln. Die Bundesdruckerei bringt dabei ihr Knowhow als Entwickler und Hersteller von hochsicheren ID-Dokumenten und -Systemen sowie das Design von Sicherheitsmerkmalen von Polycarbonatkarten ein. Der Halbleiterhersteller Infineon steuert seine Expertise bei der Entwicklung und Herstellung von kontaktlosen Sicherheits-Chips und Chipgehäusen und deren Integration in Karten bei.

Mehr Sicherheit durch Kopplung von statischer und dynamischer PIN

Die neue kontaktlose Sicherheits-Karte basiert auf der Kopplung von statischer und dynamischer PIN. Der künftige Besitzer erhält mit der Karte ein statisches Passwort, das beispielsweise aus einer Ziffernfolge bestehen kann. Bei jeder Nutzung der Karte wird zudem eine dynamische PIN-Ergänzung („One-Time Password“) abgefragt. Die dynamische PIN wird bei jedem Vorgang automatisch durch den Sicherheits-Chip in der Karte generiert und auf dem integrierten LED-Display angezeigt. Der eingesetzte Chip setzt dabei neue Maßstäbe für kontaktlose Anwendungen: Er verwendet die vom Kartenleser abgestrahlte Energie sowohl für das Betreiben des Sicherheits-Chips und die Generierung des Passworts als auch für die Stromversorgung der Displayelemente. Das LED-Display selbst ist in die Karte eingebettet, die Zahlen leuchten an der Kartenoberfläche auf.

Vorteil dieser Lösung ist die erhöhte Sicherheit: Selbst wenn die statische PIN gestohlen oder durch Schadsoftware mitgelesen würde, kann kein Unberechtigter sie nutzen, wenn die dynamische PINErgänzung fehlt. Die dynamische PIN wiederum wird nur auf der Karte erzeugt und kann vom Display nicht durch Schadsoftware mitgelesen werden.

Lösung für unterschiedlichste Kartensysteme denkbar Die Karte kann für alle Log-in-Szenarien eingesetzt werden, wie z. B. bei der Anmeldung am PC in Firmennetzwerke oder in Soziale Netzwerke im Internet. Darüber hinaus eignet sich die neue Technologie für viele weitere Kartensysteme. So könnte damit beispielsweise die Sicherheit bei der Bezahlung mit Karten im Zahlungsverkehr (EC-Karten oder Kreditkarten) erhöht werden.

Auch mobil zu nutzen

Die neuartige Polykarbonat-Chipkarte kann nicht nur mit einem herkömmlichen Lesegerät genutzt werden, sondern auch via NFCfähigen Smartphone, das als Leser fungiert.

\section{Kooperation für geschützte Sicherheitsanwendungen auf Smartphones und Tablet-PCs}

Am 21. Februar 2013 haben Symantec und Giesecke \& Devrient (G\&D), die Unterzeichnung eines Kooperationsvertrags zur Entwicklung geschützter Sicherheitsanwendungen für Smartphones und Tablet-PCs bekannt gegeben.

Im Rahmen ihrer Zusammenarbeit setzen die beiden Unternehmen auf eine hochsichere Ausführungsumgebung (Trusted Execution Environment, TEE) für sogenannte Smart Devices. Das innovative Konzept umfasst die sichere Übertragung, Speicherung und Ausführung sensibler Anwendungskomponenten und Daten in einem hardwaregeschützten Bereich auf dem Hauptprozessor von Mobilgeräten. Damit können Anwendungsentwickler ohne Einschränkung ihrer Flexibilität und Produktivität Lösungen bereitstellen, die den erhöhten Sicherheitsanforderungen gerecht werden.

Symantec wird seine Mobilanwendungen auf den neuesten Stand bringen, um die Kompatibilität zu G\&Ds „Trusted Service Manager"-Diensten (TSM) sowie der zugrundeliegenden TEE zum Schutz von kryptographischen Schlüsseln und Berechtigungsnachweisen zu gewährleisten und auf diese Weise für höchste Sicherheit zu sorgen. Die erste Anwendung dieser Art wird eine hochsichere Version der Zwei-Faktor-Authentifzierung des „Validation and ID Protection"-Services (VIP) von Symantec sein, die den Nutzern beim Zugriff auf sensible cloudbasierte Dienste zusätzliche Sicherheit bietet. Die Anwendung soll in der zweiten Jahreshälfte auf den Markt kommen. 